\title{
Subretinal angiostrongyliasis-induced optic neuritis
}

This article was published in the following Dove Press journal:

Clinical Ophthalmology

24 May 2013

Number of times this article has been viewed

\author{
Suthasinee Sinawat ${ }^{1}$ \\ Yosanan Yospaiboon' \\ Supat Sinawat ${ }^{2}$ \\ 'Vitreoretinal Unit, Department \\ of Ophthalmology, ${ }^{2}$ Department \\ of Physiology, Khon Kaen University, \\ Khon Kaen, Thailand
}

\begin{abstract}
A 27-year-old Thai male presented with progressive visual loss and a membranelike floater in the right eye that had persisted for 1 month. He had a history of eating raw foods, including snails. His initial visual acuity was counting fingers at $1 \mathrm{ft}$ and he had a relative afferent pupillary defect. A movable larva with subretinal tracks was found in the subretinal space near a normal optic disc. Visually evoked potentials showed delayed latency, which indicated secondary retrobulbar optic neuritis. A diode laser was directly applied to the motile worm. The patient was subsequently prescribed oral prednisolone and albendazole. After treatment, his visual acuity was slightly improved at $2 / 60$. Ocular manifestation is a very rare event resulting from parasitic infection. In only $1.1 \%$ of angiostrongyliasis cases is an Angiostrongylus cantonensis larva identified in the eye. Ocular angiostrongyliasis with optic neuritis may be secondary to mechanical injury and/or inflammatory reactions. Steroid treatment is recommended, although most patients have only slight visual improvement after treatment.
\end{abstract}

Keywords: Angiostrongylus cantonensis, intraocular, ocular angiostrongyliasis, parasitic infection

\section{Introduction}

Due to the habit of eating raw foods, parasitic infection does occur in many developing areas of the world. Ocular manifestation is a very rare result of parasite infection. Angiostrongylus cantonensis is the most common cause of eosinophilic meningitis. Humans are accidental hosts and become infected by ingesting third-stage larvae from raw intermediate hosts such as snails, slugs, frogs, prawns, and crabs. Infection has also rarely occurred by consuming contaminated water or uncooked vegetables. In only $1.1 \%$ of angiostrongyliasis cases is an Angiostrongylus cantonensis larva identified in the eye. ${ }^{1}$ Evidently, 35 cases of ocular angiostrongyliasis have been previously reported, ${ }^{2}$ most of which were found in Asia, especially Thailand, including the first case in the world. ${ }^{3}$ Ocular findings as a result of parasitic infection have included lateral rectus paralysis, facial palsy, uveitis, glaucoma, subretinal tracks, necrotizing retinitis, disc swelling, disc atrophy, retinal pigment alteration, and exudative retinal detachment. ${ }^{2}$ Herein, we report a case of ocular angiostrongyliasis with retrobulbar optic neuritis, in which the parasite was found in the subretinal space.

\section{Case report}

A 27-year-old Thai male presented with progressive visual loss and a membranelike floater in the right eye that had persisted for 1 month. He denied any history of headache. He had a history of eating raw foods, in particular of raw Pila sp. snails. 
Initial visual acuity of the affected eye was counting fingers at $1 \mathrm{ft}$ and he had a relative afferent pupillary defect. Inflammation was not detected in either the anterior chamber or vitreous cavity. A live roundworm, nearly $15 \mathrm{~mm}$ in length, with subretinal tracks was found near the normal optic disc. During indirect ophthalmoscopy, the larva moved rapidly to the superotemporal retina (Figure 1). A diode laser was directly applied to the parasite until it was terminated. Eosinophilia was not revealed on complete blood count. Opisthorchis viverrini eggs were found on stool examination using the concentration technique. Visually evoked potentials demonstrated a delayed latency at normal amplitude. Topical prednisolone acetate and oral prednisolone $40 \mathrm{mg}$ daily, tapered after 2 weeks, were prescribed to reduce the subsequent inflammation. Due to the patient's history of ingesting raw food, albendazole was also prescribed to eradicate any other parasitic infection. After treatment, his visual acuity was slightly improved at 2/60.

\section{Discussion}

From a review of 35 cases in the literature, the typical presenting symptom of ocular angiostrongyliasis is blurred vision without headache, although the eosinophilic meningitis may also present prior to, or coexisting with, the ocular symptoms. In some patients with angiostrongyliasis who reported having mild headache, their illness was not recognized or was misdiagnosed as migraine, tension headache, psychoneurosis, or even malaria. ${ }^{4}$ Initial visual acuity varied widely from $6 / 9$ to light perception. The incubation period is between 2 weeks and 2 months. ${ }^{1}$ Punyagupta et al

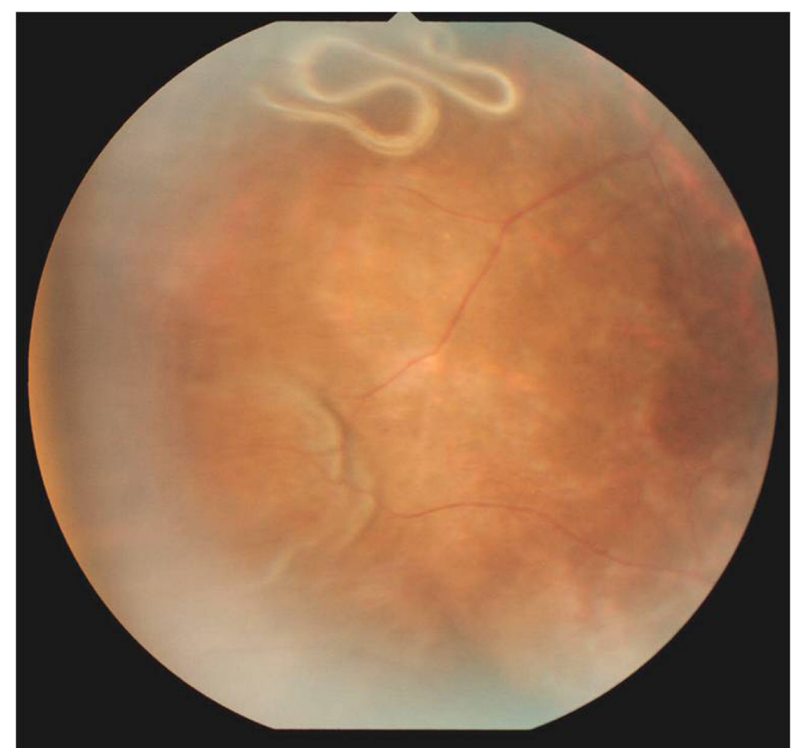

Figure I The subretinal parasite and subretinal tract at the superotemporal retina. undertook clinical studies of 484 cases of typical eosinophilic meningitis and found $16 \%$ of patients had visual impairment, while $12 \%$ had an optic disc abnormality such as papilledema or atrophy. ${ }^{4}$ In these cases, we hypothesize the intraocular parasite was not identified because no ophthalmologist was available who could perform slit-lamp ophthalmoscopy and indirect ophthalmoscopy. Three decades later, Sawanyawisuth et al conducted a 10-year review of angiostrongyliasis in the northeast of Thailand and reported that the larva could be identified in only $1.1 \%$ of patients. ${ }^{1}$ Almost all previous reports have indicated that only one living larva was found in the eye of each patient. Further, in about half of all cases, the motile worms were located in the vitreal cavity. ${ }^{2}$ We have also found that intravitreal and intracameral larvae are usually localized by fibrin, but that subretinal parasites usually move freely. ${ }^{5}$ Pathological study has indicated that $A$. cantonensis may move along the cranial nerves after reaching its fifth-stage at the brain surface. ${ }^{4}$ Cranial nerves 2,5 , and 7 have a long intracranial course, which may make them more susceptible than others. The larva can migrate to the orbit by traveling between the optic nerve and the sheath and penetrate the eye by way of the cribriform plate. A. cantonensis is easily identified by slit-lamp ophthalmoscopy and indirect ophthalmoscopy because this helminth can be easily distinguished from other parasites by its physical characteristics, so ocular examination is crucial for diagnosis. The other common intraocular roundworm is Gnathostoma spinigerum, a nematode that reaches only $4 \mathrm{~mm}$ in length and has a tapering end. Because the typical physical features of the parasite were observed and it could be treated nonsurgically, local antibody evaluation such as polymerase chain was not performed in this case report.

Although blood leukocytosis and eosinophilia have been demonstrated in $56 \%$ and $73 \%$, respectively, of eosinophilic meningitis cases, eosinophilia has not been observed in ocular angiostrongyliasis without meningitis but leukocytosis has been observed in a few cases. ${ }^{1,4}$ In cases presenting with severe headache or neurologic abnormalities, lumbar puncture indicated eosinophilic pleocytosis. ${ }^{1}$ A. cantonensis larvae and eggs have seldom been found on stool examination, while $O$. viverrini and hookworm eggs have commonly been found. ${ }^{1}$

Several treatments have been used to treat ocular angiostrongyliasis, including intravenous methylprednisolone, topical prednisolone, oral prednisolone, laser photocoagulation, and surgical removal. ${ }^{2}$ Over three decades ago in Thailand, we found that the intravitreal parasite often moved to the anterior chamber after the patient was placed in a facedown 
position. The motile worm could then be removed much more easily once in the anterior chamber. Many patients in rural and remote areas often can not be referred due to financial and transportation problems. If the intravitreal parasite moves to the anterior chamber after the patient is placed in the facedown position, general ophthalmologist can remove it. We recommend applying laser treatment before surgical removal in every case because the motile worm may disappear in the operative field. Our laser technique involved directing a laser aiming beam of 200-300 um on the pigmented gut of the larva. For a subretinal parasite, it is very important to use the laser to drive the larva away from the posterior pole, as in the case described here. In previous cases of secondary optic neuritis caused by $A$. cantonensis, most of the patients experienced only a slight improvement in visual acuity after treatment.

In previous studies, steroids have been shown to have a beneficial effect on the clinical course of ocular angiostrongyliasis. ${ }^{6}$ However, anti-helminthic drugs should be used with caution because although they have been shown effective in experimental animals, larvicidal drugs can result in serious adverse neurological effects. Cuckler et al revealed that thiabendazole treatment in experimental rats that had been infested with $A$. cantonensis for 3-4 weeks - comparable to persons with a serious infestation of this parasite - resulted in the inhibition of parasitic migration from the animals' brains. ${ }^{7}$ However, the death of many worms simultaneously in the brain or spinal cord might produce catastrophic effects from toxic substances released by the dying and necrotic worms.

Therapeutic success in ocular angiostrongyliasis mainly depends on early diagnosis and complete surgical removal of the parasite. Unfortunately, visual prognosis depends largely on the initial pathology and visual acuity; ocular angiostrongyliasis often still results in permanent visual impairment, even blindness. In the case of surgical removal, the parasitologist can clearly identify the sex and stage of the larva.

\section{Conclusion}

Many ocular angiostrongyliasis cases present with optic neuritis. Although several treatments are available and used, most patients attain only slight visual improvement following treatment. Optic neuritis can be secondary to mechanical injury due to the migrating nematodes, granulomatous inflammatory reactions due to a dead worm, or localized antigen-antibody reactions. Oral or intravenous steroid treatment is recommended for secondary optic neuritis caused by $A$. cantonensis.

\section{Disclosure}

The authors declare no conflicts of interest in this work. Written informed consent was obtained from the patient for the publication of this case report and accompanying images.

\section{References}

1. Sawanyawisuth K, Kitthaweesin K, Limpawattana P, et al. Intraocular angiostrongyliasis: clinical findings, treatments and outcomes. Trans $R$ Soc Trop Med Hyg. 2007;101(5):497-501.

2. Diao Z, Wang J, Qi H, Li X, Zheng X, Yin C. Human ocular angiostrongyliasis: a literature review. Trop Doct. 2011;41(2):76-78.

3. Ketsuwan P, Pradatsundarasar A. Second case of ocular angiostrongyliasis in Thailand. Am J Trop Med Hyg. Jan 1966;15(1):50-51.

4. Punyagupta S, Juttijudata $P$, Bunnag T. Eosinophilic meningitis in Thailand. Clinical studies of 484 typical cases probably caused by Angiostrongylus cantonensis. Am J Trop Med Hyg. 1975;24(6 Pt 1): 921-931.

5. Sinawat S, Sanguansak T, Angkawinijwong T, et al. Ocular angiostrongyliasis: clinical study of three cases. Eye (Lond). 2008; 22(11):1446-1448.

6. Sawanyawisuth K, Sawanyawisuth K. Treatment of angiostrongyliasis. Trans R Soc Trop Med Hyg. 2008;102(10):990-996.

7. Cuckler AC, Egerton JR, Alicata JE. Therapeutic effect of thiabendazole on Angiostrongylus cantonensis infections in rats. J Parasitol. 1965; 51(3):392-396.
Clinical Ophthalmology

\section{Publish your work in this journal}

Clinical Ophthalmology is an international, peer-reviewed journal covering all subspecialties within ophthalmology. Key topics include: Optometry; Visual science; Pharmacology and drug therapy in eye diseases; Basic Sciences; Primary and Secondary eye care; Patient Safety and Quality of Care Improvements. This journal is indexed on

\section{Dovepress}

PubMed Central and CAS, and is the official journal of The Society of Clinical Ophthalmology (SCO). The manuscript management system is completely online and includes a very quick and fair peer-review system, which is all easy to use. Visit http://www.dovepress.com/ testimonials.php to read real quotes from published authors. 